\title{
Investigation on relationship between epicentral distance and growth curve of initial P-wave propagating in local heterogeneous media for earthquake early warning system
}

\author{
Kyosuke Okamoto ${ }^{*}$ and Seiji Tsuno
}

\begin{abstract}
In the earthquake early warning (EEW) system, the epicenter location and magnitude of earthquakes are estimated using the amplitude growth rate of initial P-waves. It has been empirically pointed out that the growth rate becomes smaller as epicentral distance becomes far regardless of the magnitude of earthquakes. So, the epicentral distance can be estimated from the growth rate using this empirical relationship. However, the growth rates calculated from different earthquakes at the same epicentral distance mark considerably different values from each other. Sometimes the growth rates of earthquakes having the same epicentral distance vary by $10^{4}$ times. Qualitatively, it has been considered that the gap in the growth rates is due to differences in the local heterogeneities that the P-waves propagate through. In this study, we demonstrate theoretically how local heterogeneities in the subsurface disturb the relationship between the growth rate and the epicentral distance. Firstly, we calculate seismic scattered waves in a heterogeneous medium. First-ordered PP, PS, SP, and SS scatterings are considered. The correlation distance of the heterogeneities and fractional fluctuation of elastic parameters control the heterogeneous conditions for the calculation. From the synthesized waves, the growth rate of the initial P-wave is obtained. As a result, we find that a parameter (in this study, correlation distance) controlling heterogeneities plays a key role in the magnitude of the fluctuation of the growth rate. Then, we calculate the regional correlation distances in Japan that can account for the fluctuation of the growth rate of real earthquakes from 1997 to 2011 observed by K-NET and KiK-net. As a result, the spatial distribution of the correlation distance shows locality. So, it is revealed that the growth rates fluctuate according to the locality. When this local fluctuation is taken into account, the accuracy of the estimation of epicentral distances from initial P-waves can improve, which will in turn improve the accuracy of the EEW system.
\end{abstract}

\section{Findings}

\section{Introduction}

In the earthquake early warning (EEW) system, the magnitude and epicenter location of earthquakes are estimated within several seconds after P-wave arrival. Nakamura (1988) developed an EEW system called the Urgent Earthquake Detection and Alarm System (UrEDAS), used by railways to stop trains in advance of the strong seismic motion. In the UrEDAS system, the magnitude of earthquakes is estimated from the predominant frequency of P-waves using a single-station record (the

\footnotetext{
* Correspondence: okamoto.kyosuke.51@rtri.or.jp

Railway Technical Research Institute, 2-8-38 Hikari-cho, Kokubunji-shi, Tokyo 185-8540, Japan
}

single-station method). Then, the epicentral distance is estimated using the estimated magnitude and the observed amplitude of the P-wave. Odaka (2003) suggested a new method to estimate epicentral distance also using a single-station record. In this method, Eq. (1) is fitted to the early portion of the P-wave envelopes.

$$
B t \exp (-A t)
$$

where $A$ and $B$ are constants, and $t$ represents time. $A$ and $B$ are determined by a least-squares method. From the $B$ value, epicentral distances are estimated using an empirical relationship. Odaka (2003) pointed out that the $B$ value decreases as the epicentral distance increases. The decrease in the $B$ value is mainly due to the

\section{Springer Sprin}

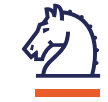

(c) 2015 Okamoto and Tsuno. Open Access This article is distributed under the terms of the Creative Commons Attribution 4.0 International License (http://creativecommons.org/licenses/by/4.0/), which permits unrestricted use, distribution, and reproduction in any medium, provided you give appropriate credit to the original author(s) and the source, provide a link to the Creative Commons license, and indicate if changes were made. 
geometrical spreading and scattering and anelastic attenuation of seismic waves. As the amplitude of P-waves decreases by propagating through the medium, the amplitude growth rate of the early portion of the P-waves also decreases. The decrease seemed to be independent of the magnitude of the earthquakes. Thus, the $B$ value can be used to estimate the epicentral distance. However, in practice, the $B$ values of different earthquakes show quite different values even if the epicentral distances are same. The gap in the $B$ values is sometimes by $10^{4}$ times. So, the epicentral distances estimated from $B$ values include error to some extent, sometimes the error reaches to $10^{2} \mathrm{~km}$ order. Possible causes of the gap in the $B$ values are differences in the radiation pattern, source function, conditions of the subsurface, etc. However, Odaka (2003) pointed out that differences in radiation patterns do not result in an order-of-magnitude difference of the $B$ values, except for the case when the seismic station is located near the nodal plane. Moreover, the $B$ values of the observed earthquakes in this study are calculated from high-frequency (higher than $10 \mathrm{~Hz}$ ) seismic waves. It has been pointed out that the radiation pattern of high-frequency seismic waves (higher than several hertz) seems to be isotropic (e.g., Liu and Helmberger 1985 for S-wave and Kobayashi et al. 2014 for P-wave). Source functions whose duration times reach several seconds or more, i.e., earthquakes bigger than magnitude $M 7$, may cause a significant change to the $B$ value. This is because the source duration time is longer than the length of the time window to calculate the $B$ value, which is $2 \mathrm{~s}$ in the present EEW system. However, the $B$ value fluctuates considerably even when the magnitude of earthquakes is relatively small, like M4 or M5. In that case, the source duration time is on the order of around $10^{-2}-10^{-1} \mathrm{~s}$. So, the source function, especially the source duration time, cannot be the main cause for the $B$ value fluctuation, especially for relatively low-magnitude earthquakes. The remaining potential cause for the $B$ value fluctuation is seismic attenuation and scattering due to heterogeneities in the subsurface. Tsukada et al. (2004) conducted a theoretical calculation on the broadening of scalar wave envelopes based on three-dimensional von Kármán type random medium. They found that the gap in the $B$ values of different earthquakes having the same epicentral distance can be attributed to differences in the scattering condition. In their study, it is assumed that conversion waves between P and S waves (i.e., PS and SP conversions) are negligible. However, $\mathrm{P}$ coda waves seem to be dominated by the SP conversion waves (Yoshimoto et al. 1997a).

In this study, we theoretically calculated P-waves in a heterogeneous medium considering the conversion waves between $\mathrm{P}$ and $\mathrm{S}$ waves and obtained theoretical $B$ values. We showed that variation in the condition of heterogeneities (correlation distance) changes the $B$ values and causes fluctuation in the $B$ values even if the epicentral distances are the same. Then, we compared the theoretically calculated $B$ values with those calculated from earthquakes in 1996-2011 observed by K-NET and KiK-net in Japan. The $B$ values calculated from various earthquakes showed different values even when their epicentral distances were the same. We calculated regional parameters of heterogeneities (correlation distances) in Japan that can account for the fluctuation in the observed $B$ values. As a result, it was found that the spatial distribution of the correlation distances showed a similar tendency with that of parameters reflecting local heterogeneities, like coda- $Q$. The observed $B$ values also showed similar distribution to that of the correlation distance.

In this study, we hypothesize that the variation in the condition of heterogeneities causes significant $B$ value fluctuation, and verify this hypothesis. The fluctuation in the $B$ values causes error in estimation of epicentral distances. Thus, if we address the fluctuation in $B$ values by considering the local condition of heterogeneities, the estimation of epicentral distances becomes more accurate, which in turn improves the accuracy of the EEW system.

\section{Calculation of theoretical P-wave}

We calculated theoretical P-waves and its scattering waves (PP, PS, SP, and SS conversions) using the method by Sato (1984). P-waves were calculated on the basis of the first-ordered Born approximation. The P-wave radiating from a source fault in the subsurface is scattered by heterogeneous structures, and the P- and scattered waves are observed at a receiver on the surface. The heterogeneous structure is characterized by an exponential autocorrelation function with the fractional fluctuation $\varepsilon$ and the correlation distance $a$. Gao et al. (1983) and Hoshiba (1991) pointed out that the first-ordered Born approximation is appropriate within a time window whose length is shorter than the mean free time of the medium by about 0.57 times. When P-wave velocity is $5.0 \mathrm{~km} / \mathrm{s}$ and the mean free path is $10^{2}-10^{3} \mathrm{~km}$, the time appropriate to the first-ordered Born approximation is $10^{1}-10^{2} \mathrm{~s}$ from the origin of earthquakes, which corresponds to hypocentral distance of $\sim 10^{2} \mathrm{~km}$. Frankel and Clayton (1986) numerically showed that the amplitude growth of P-waves is well accounted for by the firstordered Born approximation when $\varepsilon$ is less than $10 \%$. In this study, we used a 2-s length time window from the onset of P-waves, and considered several $\%$ of $\varepsilon$, so that we could ignore multiple scatterings. Source faults have parameters, which are strike, dip-and-slip angles, depth, epicentral distance, and moment magnitude, and these are all considered in the theoretical calculation. So, the geometry of source-receiver configuration, the size, and 
the radiation pattern of earthquakes are considered in the theoretical calculation. Though the radiation pattern is taken into account for the theoretical calculation, the directivity effect of earthquakes is not considered because we adopt a point source. The source function we adopted is the $\omega$-squared model. The calculated waves for a given frequency are velocity envelopes, and simply multiplying the angular frequency $\omega$ to the velocity envelopes, we get the acceleration envelope waveforms. Waveforms were calculated for $4-20 \mathrm{~Hz}$ at $1-\mathrm{Hz}$ intervals, and the waves are converted to the time-domain by the Fourier transform.

\section{Example of theoretical calculation}

Figure 1 shows a synthesized P-wave envelope and an observed seismogram, both are UD components of acceleration. The analyzed earthquake, which occurred on May 8, 2005 at Tochigi prefecture, Japan, had a moment magnitude of 4.5 and focal depth of $9.7 \mathrm{~km}$. The observed seismogram was recorded at the K-NET SIT008 station. The observed seismogram was filtered by a
Butterworth band-pass filter $(4-20 \mathrm{~Hz})$. When the theoretical P-wave envelope was calculated, $\mathrm{P}$ - and $\mathrm{S}$-wave velocities $\left(V_{p}=5,515 \mathrm{~m} / \mathrm{s}, V_{s}=3,126 \mathrm{~m} / \mathrm{s}\right)$ were taken from Matsubara and Obara (2011), and the focal mechanism and location were given from the JMA unified earthquake catalog. The velocities at the intermediate point of the hypocenter and the station were used. The density was calculated from the P-wave velocity using the equation based on Gardner's relationship (Gardner et al. 1974). In the original Gardner's relationship, density is in proportion to the forth root of P-waves. In this study, we used a modified Gardner's relationship shown by Eq. (2).

$$
\rho=1.5 V_{p}^{0.35}
$$

Equation (2) was obtained from the core samples of the boreholes down to the depth of the seismic bedrock in Chiba prefecture (Chiba prefecture 2004). Here, $\rho=$ $2.73 \mathrm{~g} / \mathrm{cm}^{3}$ was obtained from Eq. (2). The heterogeneous medium was characterized by $a=800 \mathrm{~m}$ and $\varepsilon=$ $8 \%$. The theoretical $\mathrm{P}$-wave envelope matched the

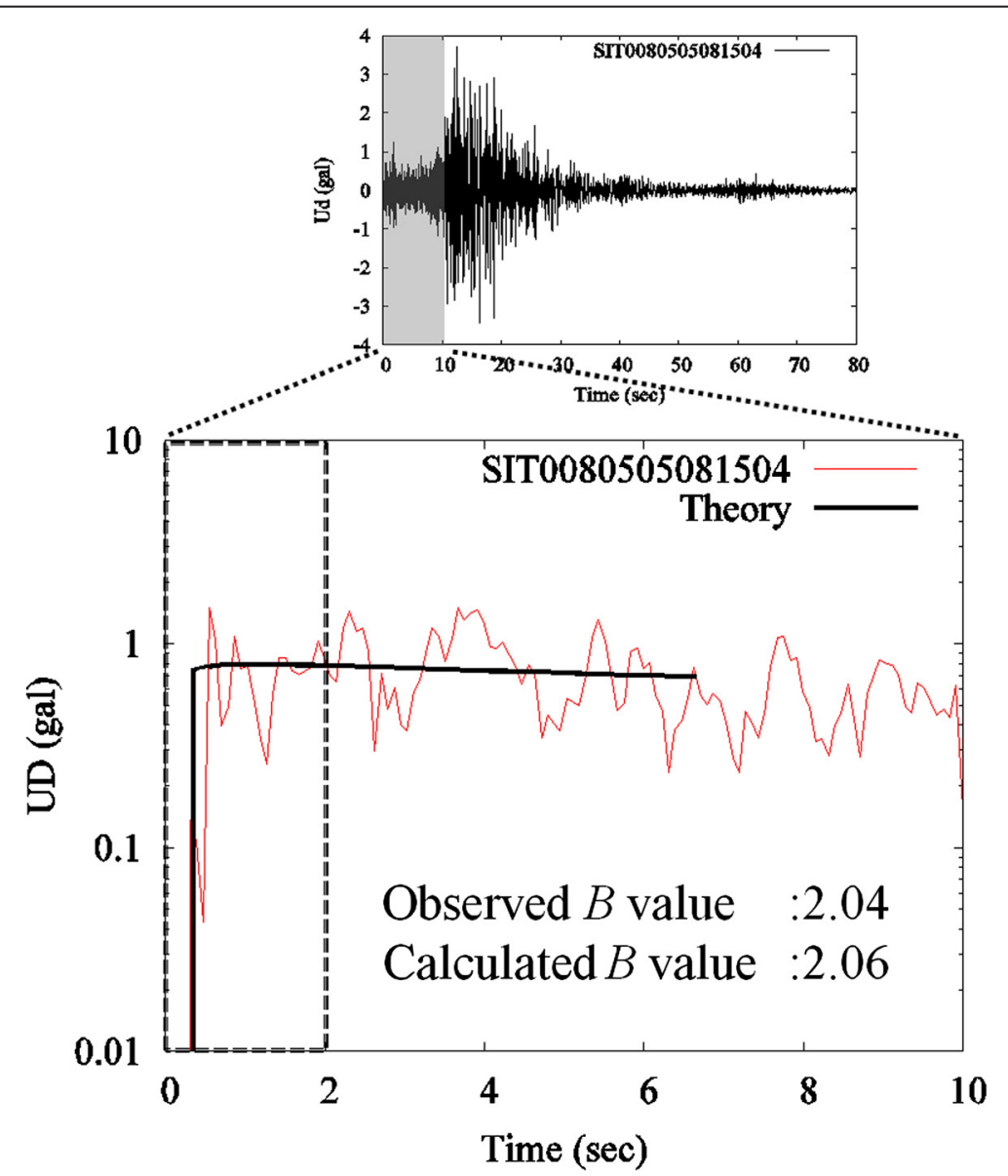

Fig. 1 Observed and synthesized envelope. Observed seismogram is shown by the thin line and synthesized envelope is shown by the bold line. The gray shadow shows the 2 -s time window which is used to calculate the $B$ value 
observed seismogram well. Thus, P-wave envelopes are calculated, considering the focal mechanism, location, and magnitude of earthquakes and the parameters $\left(V_{p}\right.$, $\left.V_{s}, \rho, a, \varepsilon\right)$ of the medium that the seismic waves propagate through. Those parameters, except $a$ and $\varepsilon$, are determined when the focal mechanism and location of the hypocenter and the station are given. The theoretical $B$ value is obtained by fitting Eq. (1) to the theoretical envelope. To fit Eq. (1), a least-squares method is used for a 2-s envelope from the onset of the P-wave.

\section{Comparison of theoretical and observed $B$ values}

We calculated the $B$ value from the UD component of the observed acceleration waveform envelopes. The waveforms were observed by the K-NET and KiK-net stations. The observation term was from March 1996 to April 2011, and the magnitude range of the analyzed earthquakes was from 4.0 to 5.0. The source duration time was far shorter than the time window length $(2 \mathrm{~s})$. The total number of analyzed earthquakes and seismograms were 55 and 2373 (the geometry is shown in Fig. 2). The arrival time of P-waves was identified by the Short Time Average to Long Time Average (STA/ LTA) method (Horiuchi et al. 2005). A band-pass filter $(10-20 \mathrm{~Hz})$ was applied to the seismogram. Then, the envelope was synthesized from the band-passed seismogram by taking the absolute value and smoothing it with $10-\mathrm{Hz}$ intervals. Finally, the $B$ value was acquired by fitting Eq. (1) to the envelope using a least-squares method. A 2-s envelope from the P-wave arrival was used for the fitting. We call the $B$ value obtained from the real earthquakes the observed $B$ value $\left(B_{\text {obs. }}\right)$. On the other hand, the $B$ value was also calculated from the theoretically synthesized acceleration envelopes. We call it the theoretical $B$ value $\left(B_{\text {theo. }}\right)$. Focal mechanisms, P- and $\mathrm{S}$-wave velocities and density are given for the theoretical calculation as described in the former chapter. The remaining parameters required for the theoretical calculation are fractional fluctuation $\varepsilon$ and correlation distance $a$. We fixed $\varepsilon$ to $6 \%$ and $a$ to $150,200,600$, or $1000 \mathrm{~m}$. The

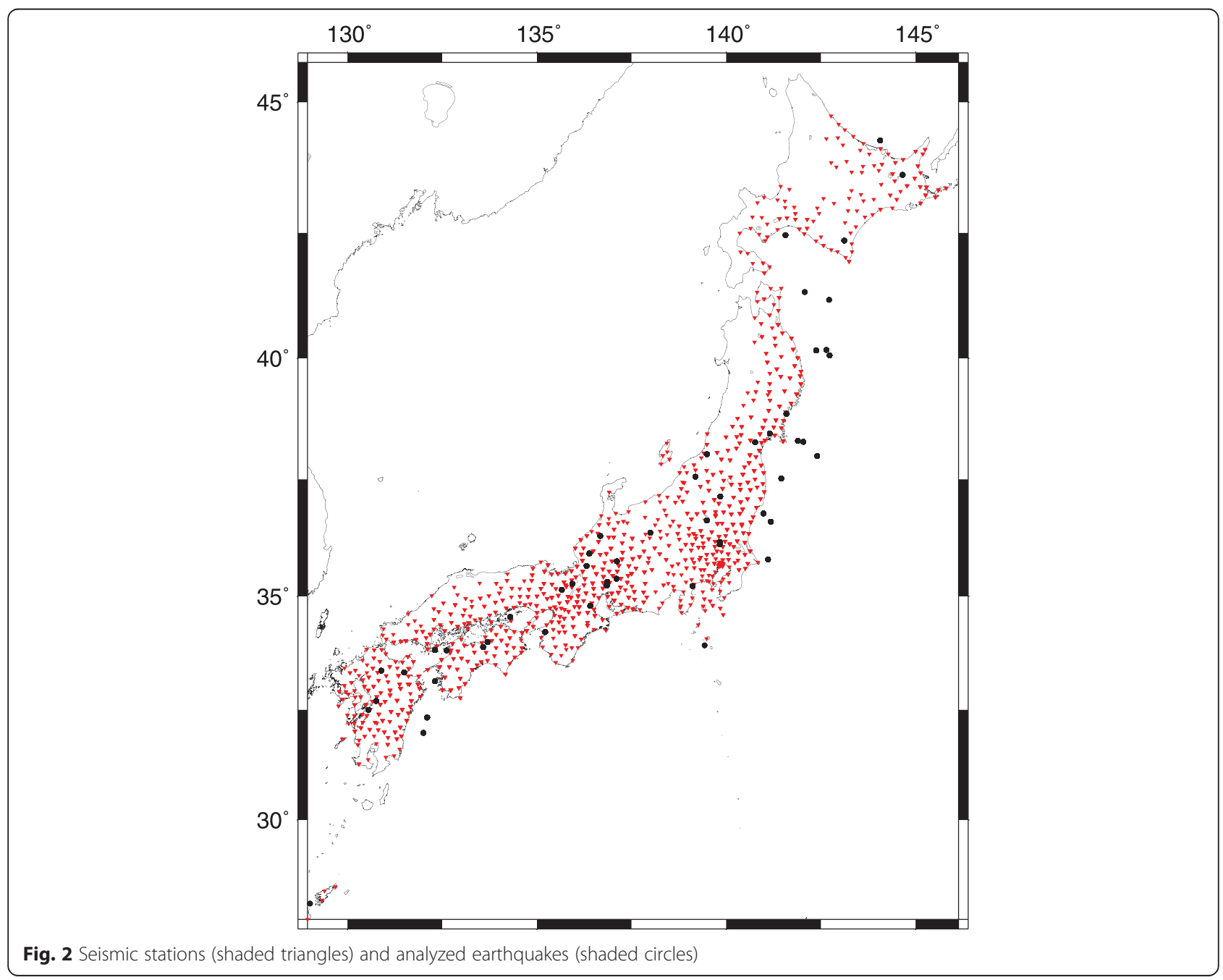


values of those parameters are comparable with those reported by Yoshimoto et al. (1997b).

Figure 3 shows the $B_{o b s .}$ and $B_{\text {theo. }}$ values for the analyzed earthquakes. The $B_{o b s}$. values from different earthquakes having the same epicentral distance vary on an order of more than $10^{4}$. The $B_{\text {theo. }}$ values were calculated for each analyzed earthquake. The four lines $(a=150$, 200,600 , and $1000 \mathrm{~m}$ ) of the $B_{\text {theo. }}$ values covered the wide distribution of the $B_{o b s}$ values. The shorter the correlation distance is, the smaller value the $B_{\text {theo }}$ marks. Thus, the fluctuation of the $B_{o b s}$. values is accounted for by variation in $a$. When $a$ is fixed, the stretch of the $B_{\text {theo. }}$ values at a given epicentral distance is far smaller than that of $B_{\text {obs. }}$ values. At that time, the stretch of the $B_{\text {theo. }}$ values is due to variation in the focal mechanism, the location and size of the earthquakes, and elastic parameters of the medium. The fluctuation of the $B_{o b s}$. values could not be accounted for only by the changes in those parameters. Consequently, variation in $a$ is a major cause for the fluctuation in the $B_{o b s}$. values. The four lines account for the wide distribution of the $B_{\text {obs. }}$. values well.

At each K-NET and KiK-net station in Fig. 2, we took the average of $\log \left(B_{\text {obs. }} / B_{\text {theo. }}\right)$ over all analyzed earthquakes detectable at the station, changing $a$ to 150,200 , 600 , and $1000 \mathrm{~m}$. Then, the value of $a$ which gives the minimum value of the average of $\log \left(B_{\text {obs. }} / B_{\text {theo. }}\right)$ was determined for each station (Fig. 4). Figure $5 \mathrm{a}$, b show the average of $\log \left(B_{\text {obs. }} / B_{\text {theo. }}\right)$ before and after the value of $a$ is optimized. Value of $a$ at each station is fixed to $200 \mathrm{~m}$ in Fig. $5 \mathrm{a}$, and the value of $a$ is selected to minimize the average of $\log \left(B_{\text {obs. }} / B_{\text {theo. }}\right)$ in Fig. $5 \mathrm{~b}$. The average of $\log (-$ $\left.B_{\text {obs. }} / B_{\text {theo. }}\right)$ in Fig. $5 \mathrm{~b}$ is nearer to 0.0 than that in Fig. $5 \mathrm{a}$. The value of $a$ controls heterogeneities, e.g., conditions

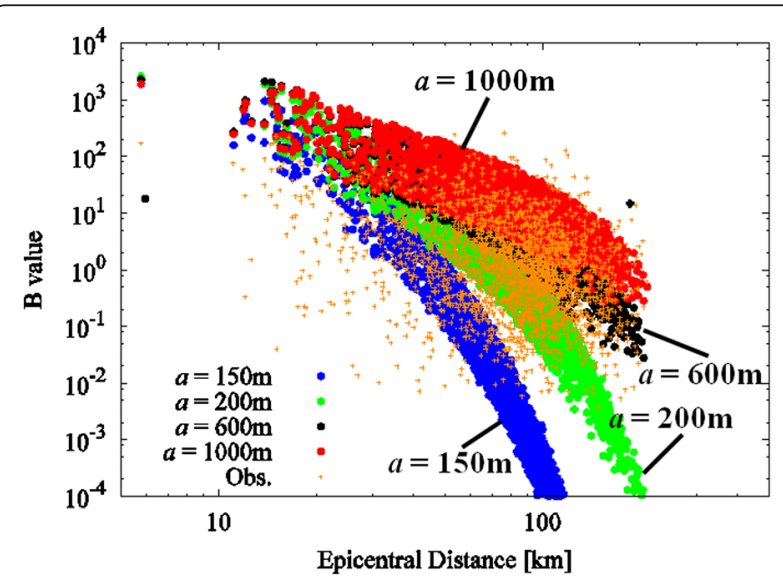

Fig. $3 B_{o b s .}$ and $B_{\text {theo. }}$ values against epicentral distance. $B_{\text {obs. }}$ values are shown by small dots ( ). $B_{\text {theo. }}$ values are calculated using the parameters (fault parameters and medium parameters) of target earthquakes. The red circles are results of $a=1000 \mathrm{~m}$, black $600 \mathrm{~m}$, green $200 \mathrm{~m}$, and blue $150 \mathrm{~m}$ of seismic scattering, absorption, etc. So, it changes the shape of the seismic wave envelopes. When $a$ is fixed to $200 \mathrm{~m}$ over whole of Japan, the average of $\log \left(B_{\text {obs. }}\right.$. $B_{\text {theo. }}$ ) is a proxy of the difference between the given condition of heterogeneities $(a=200 \mathrm{~m})$ and the real one. At that time, the spatial distribution of the average of $\log \left(B_{\text {obs. }} / B_{\text {theo. }}\right)$ (Fig. $\left.5 \mathrm{a}\right)$ shows a negative correlation with that of coda- $Q^{-1}\left(Q_{c}^{-1}\right)$ value $(8-16 \mathrm{~Hz})$ calculated in the preceding studies (e.g., Carcole and Haruo 2010; Jin and Aki, 2005) especially near the Kanto and Tohoku regions. The side of the Pacific Ocean of those regions, the average of $\log \left(B_{\text {obs. }} / B_{\text {theo. }}\right)$ in Fig. $5 \mathrm{a}$, is relatively high while $Q_{c}^{-1}$ from Carcole and Sata (2010) relatively low. $Q_{c}^{-1}$ indicates the energy loss of seismic waves due to heterogeneities and reflects the heterogeneous conditions of the medium. For example, Okamoto et al. (2013) pointed out that $Q_{c}^{-1}$ has a relationship with the density of cracks in medium. When the $Q_{c}^{-1}$ is high, seismic attenuation is also high. The correlation between the average of $\log \left(B_{\text {obs. }} / B_{\text {theo. }}\right)$ and the value of $Q_{c}^{-1}$ also supports our hypothesis that the $B_{o b s}$. values vary due to local variation in the condition of heterogeneities.

\section{Locality of observed $B$ value}

The K-NET and KiK-net stations are divided into the four groups (Fig. 4) in terms of the station's $a$ value $(150,200,600$, or $1500 \mathrm{~m})$. The value of $a$ at a station was chosen to minimize the average of $\log \left(B_{\text {obs. }} / B_{\text {theo. }}\right)$ over all the seismograms recorded by the station in the former chapter. To evaluate the respective $B_{o b s}$. values of all the analyzed earthquakes, the $B_{\text {obs. }}$ values are plotted, with colors indicating the four groups (Fig. 6). The $B_{o b s}$. values are clearly divided into four clusters. Higher $B_{\text {obs }}$. values are obtained at the stations characterized by longer $a$. The $B_{o b s .}$ value has locality, which is derived from the locality of the $a$ value. The lines in Fig. 6 are approximate curves for each cluster of the $B_{o b s}$. values. The approximate curves are obtained from the analogy of the distance decay equation of seismic wave amplitude. The equation of the approximate curves is Eq. (3). Hereafter, we call this equation as $B-\Delta$ equation.

$$
\log B=\alpha \log \Delta+\beta+\gamma \cdot \Delta
$$

where $\Delta$ is epicentral distance, $\alpha, \beta$, and $\gamma$ are constants. $\alpha$ relates mainly to geometrical spreading, $\beta$ to static effect especially around source and receiver because it is not multiplied by $\Delta$, and $\gamma$ to intrinsic and scattering attenuations along path. At first, we deter-

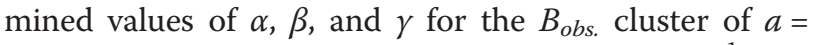
$1000 \mathrm{~m}$ by grid search. $\alpha, \beta$, and $\gamma$ are $-4.40 \times 10^{-1}, 3.50$ and $-2.23 \times 10^{-2}$. Then, we determine those values for the $B_{o b s}$. clusters of $a=600,200$, and $150 \mathrm{~m}$. At that time, $\alpha$ and $\beta$ are fixed to the values of the $B_{o b s .}$ cluster 


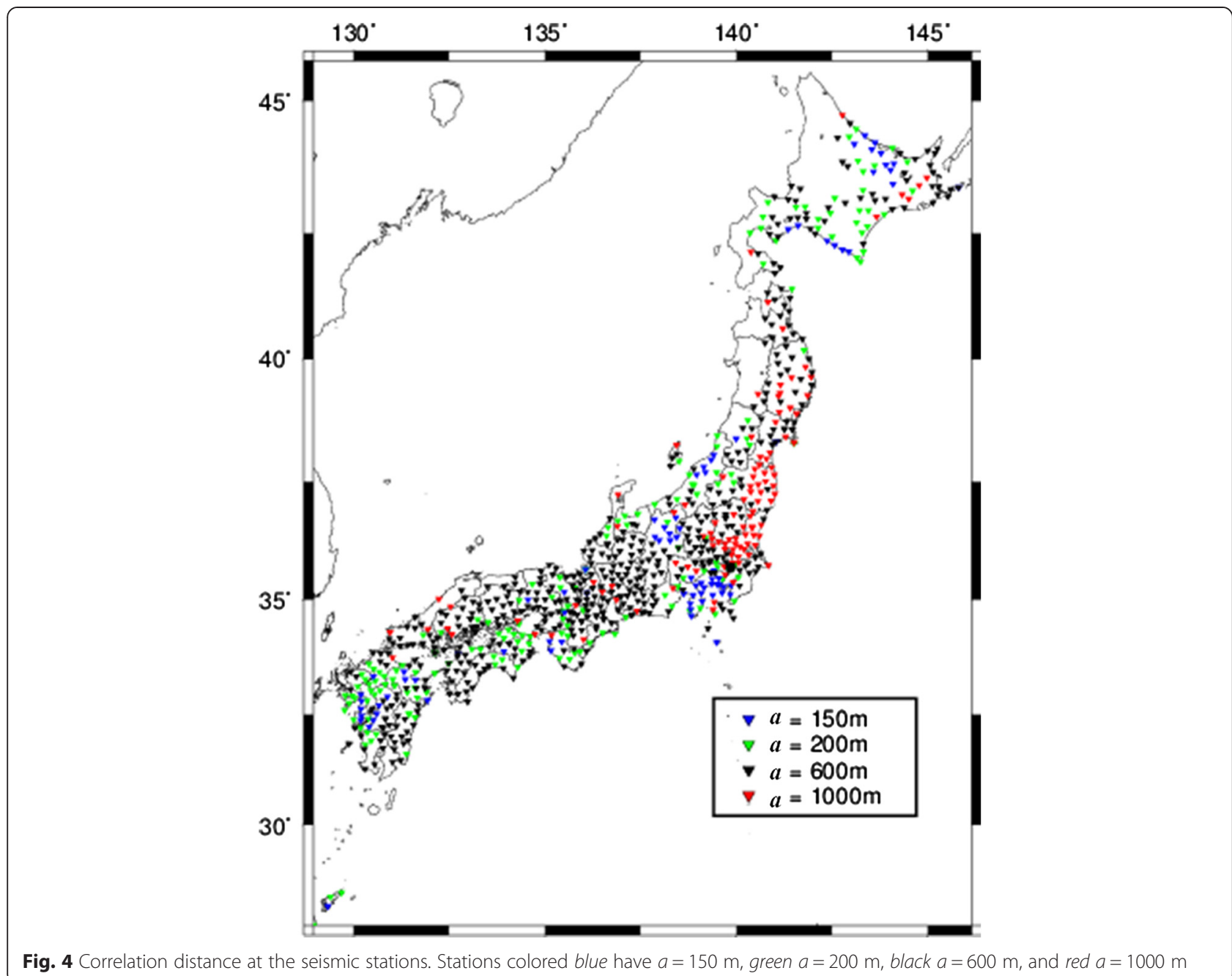

of $a=1000 \mathrm{~m}$; that is, $\alpha=-4.40 \times 10^{-1}$ and $\beta=3.50$. Only $\gamma$, which controls intrinsic and scattering attenuations, is changed, since we focus on those effects. The value of $\gamma$ is determined by a grid search. In this procedure, the differences among the $B_{o b s}$. clusters are attributed to local changes in the intrinsic and scattering attenuations, $\gamma$. Table 1 shows $\gamma$ values for each $a$ value.

The shorter the correlation distance is, the higher the observed attenuation is due to heterogeneities. Here, higher attenuation corresponds to higher absolute value of $\gamma$. In the areas where correlation distance is short, the initial P-wave amplitude grows more gradually because the energy in the incident wave scatters into the subsequent wave trains and is absorbed. From the gradually growing initial $\mathrm{P}$-waves, smaller $B$ values are observed. On the other hand, higher $B$ values are observed at the area where the correlation distance is longer. It is because the incident wave is not scattered so much and has more energy in its initial part. So, the initial P-wave amplitude grows rapidly.
In the conventional EEW method, especially for railways, the $B-\Delta$ equation has the term of $\alpha$ only, which is related to geometrical spreading mainly. Moreover, the coefficient $\alpha$ is calculated using all the $B_{o b s}$. values. The $B_{o b s}$. values at a given epicentral distance have a considerable extent of variance (sometimes $10^{4}$ times) due to difference in heterogeneous conditions as we showed. So, the epicentral distances estimated by the $B-\Delta$ equation also have errors. The errors in the estimated epicentral distances can be on the order of $10^{2} \mathrm{~km}$. The errors decrease the accuracy of the EEW system. When the $B$ $\Delta$ equation is defined considering local heterogeneities properly, the errors in the estimated epicentral distances will decrease.

We propose a method to consider the local heterogeneities, that is, changing the $\gamma$ value in Eq. (3) according to the station's $a$ value. The $\gamma$ value of the target $a$ value was calculated using the $B_{\text {obs. }}$ values obtained at the station characterized by the target $a$ value. Other choices to consider the local heterogeneities are changing the $\beta$ 


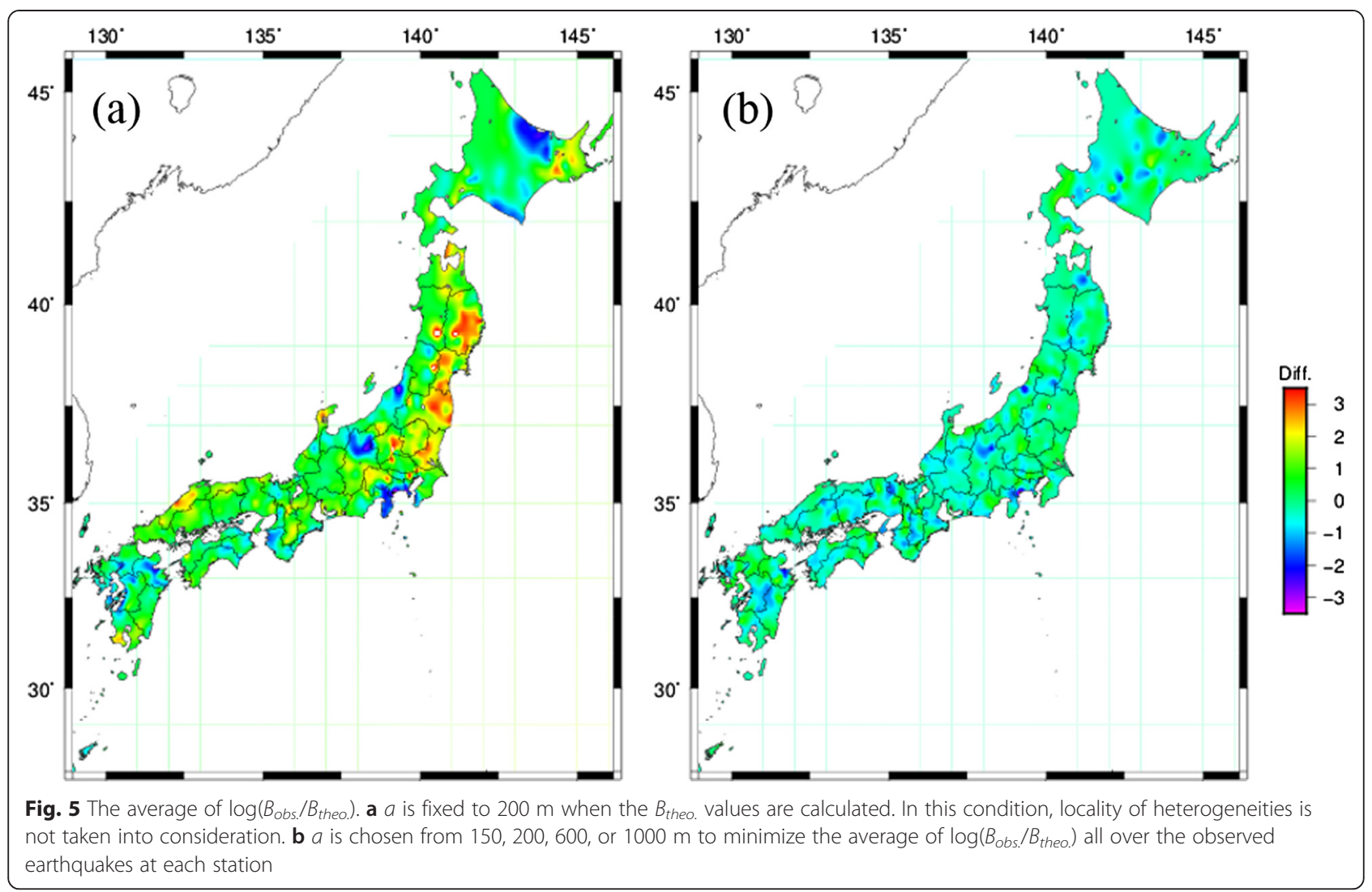

value or both the $\beta$ and $\gamma$ values in Eq. (3). The $\beta$ value represents effects around source and receiver, and the $\gamma$ value represents effects along path. In this study, although we determined the $\gamma$ values according to the station's $a$ value, the determination of the $\gamma$ values according to the geological conditions (e.g., kind of

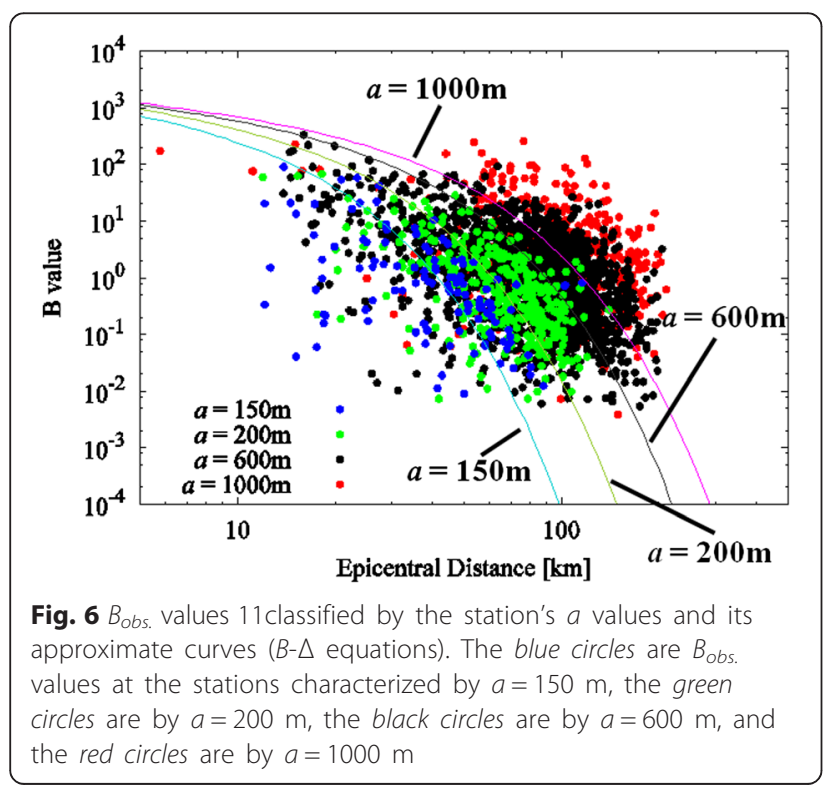

plates, tectonic line) could be a more simple choice in practical use.

\section{Summary and discussion}

In this study, we hypothesized that $B$ values for a given epicentral distance fluctuate strongly due to differences in local heterogeneous conditions. Firstly, we theoretically calculated $B$ values for earthquakes occurring in March 1996 to April 2011. In this calculation, we chose parameters controlling the conditions of heterogeneities, that is, the correlation distance $a$ and fractional fluctuation $\varepsilon$, to account for the observed $B$ values. As a result, we found that differences in the $a$ value are one of the major causes that make the $B$ values fluctuate. The spatial distribution of the $a$ value has a similar tendency with that of $Q_{c}^{-1}$, which is said to have a correlation with heterogeneities in the subsurface. This means that the

Table 1 Relation between $a$ and $\gamma$ values

\begin{tabular}{ll}
\hline$a$ value & $\gamma$ value \\
\hline $150 \mathrm{~m}$ & $-6.71 \times 10^{-1}$ \\
$200 \mathrm{~m}$ & $-4.42 \times 10^{-1}$ \\
$600 \mathrm{~m}$ & $-2.94 \times 10^{-1}$ \\
$1000 \mathrm{~m}$ & $-2.23 \times 10^{-1}$ \\
\hline
\end{tabular}


observed $B$ values have locality according to the local heterogeneities.

Currently in the EEW system, epicentral distances are estimated from the empirical relationship with $B$ values ( $B-\Delta$ equation), which contains errors due to heterogeneities in the subsurface. Although the $B$ values for a given epicentral distance fluctuate very much, the empirical relationship is based on the assumption that the $B$ values decrease against epicentral distances monotonically. Thus, the epicentral distance estimated by the empirical relationship has a considerable error, sometimes on the order of $10^{2} \mathrm{~km}$. As a result of our study, we found that the distribution of $B$ values shows tendencies according to the regional heterogeneities. So that, by considering local conditions of heterogeneities, the accuracy of epicentral distance estimation will be improved. In this study, we proposed that the $B-\Delta$ equation optimized by the station's $a$ value could improve the estimation accuracy.

The estimation is done by a single station. An advantage of the single-station method is that information on earthquakes is quickly obtained. This advantage is especially effective for warning high-speed railways, like the Shinkanse, which need longer lead time, to decrease its speed as much as possible before strong motions hit trains. Recently, an approach for improving the accuracy of back-azimuth estimation to estimate epicentral direction was studied (Noda et al., 2012). So, both epicentral distances and directions can be obtained more accurately. The accuracy of the EEW system will be improved using those new techniques.

\section{Competing interests}

The authors declare that they have no competing interests.

\section{Authors' contributions}

$\mathrm{KO}$ carried out theoretical calculations of seismic waves, analysis and interpretation of observed earthquakes, and drafted the manuscript. ST participated in the design of the study, carried out the interpretation, and was involved in revising the manuscript. All authors read and approved the final manuscript.

\section{Acknowledgements}

We would like to thank the National Research Institute for Earth Science and Disaster Prevention (NIED) for making K-NET and KiK-net data available and the Japan Meteorological Agency (JMA) for making the JMA unified earthquake catalog available. Some of the figures were drawn by Generic Mapping Tools (GMT) software.

Received: 1 June 2015 Accepted: 6 October 2015

Published online: 13 October 2015

\section{References}

Carcole E, Haruo S (2010) Spatial distribution of scattering loss and intrinsic absorption of short-period $\mathrm{S}$ waves in the lithosphere of Japan on the basis of the multiple lapse time window analysis of Hi-net data. Geophys J Int 180:268-290. doi:10.1111/j.1365-246X.2009.04394.x

Chiba prefecture (2004) Subsurface survey report of Chiba prefecture in 2004 (CD-ROM), Chiba prefecture (in Japanese)
Frankel A, Clayton RW (1986) Finite difference simulations of seismic scattering: implication for the propagation of short-period seismic waves in the crust and models of crustal heterogeneity. J Geophys Res 91:6465-6489

Gao LS, Biswas NN, Lee LC, Aki K (1983) Effects of multiple scattering on coda waves in three-dimensional medium. Pageoph 121:3-15

Gardner GHF, Gardner LW, Gregory AR (1974) Formation velocity and density—-The diagnostic basics for stratigraphic traps. Geophysics 39:770-780

Horiuchi S, Negishi H, Abe K, Kamimura A, Fujinawa Y (2005) An automatic processing system for broadcasting earthquake alarms. Bull Seismol Soc Am 95:708-718. doi:10.1785/0120030133

Hoshiba M (1991) Simulation of multiple-scattered coda wave excitation based on the energy conservation law. Phys Earth Planet Inter 67:123-136

Liu H-L, Helmberger DV (1985) The 23:19 aftershock of the 15 october 1979 imperial valley earthquake: more evidence for an asperity. Bull Seismol Soc Am 75:689-708

Jin A, Aki K (2005) High-resolution maps of Coda Q in Japan and their interpretation by the brittle-ductile interaction hypothesis. Earth Planet Space 57:403-409

Kobayashi M, Takemura S, Yoshimoto K (2014) Distortion of the apparent P-wave radiation pattern. In: Abstracts of the seismological society of Japan 2014, Fall meeting. Niigata convention center, Niigata, pp 24-26, November 2014 (in Japanese)

Matsubara M, Obara K (2011) The 2011 off the Pacific coast of Tohoku Earthquake related to a strong velocity gradient with the Pacific plate. Earth Planet Space 63:663-667. doi:10.5047/eps.2011.05.018

Nakamura Y (1988) On the urgent earthquake detection and alarm system (UrEDAS). In: Proceedings of Ninth World Conference on Earthquake Engineering, Tokyo-Kyoto, Japan, 2-9 August 1988

Noda S, Yamamoto S, Sato S, Iwata N, Korenaga M, Ashiya K (2012) Improvement of back-azimuth estimation in real-time by using a single station record. Earth Planet Space 64:305-308. doi:10.5047/eps.2011.10.005

Odaka T, Ashiya K, Tsukada S, Sato S, Ohtake K, Nozaka D (2003) A new method of quickly estimating epicentral distance and magnitude from a single seismic record. Bull Seismol Soc Am 93:526-532

Okamoto K, Mikada H, Goto T, Takekawa J (2013) Numerical analysis of the relationship between time-variant coda- $\mathrm{Q}$ and the variation in crustal stress. Geophys J Int 195:575-581. doi:10.1093/gji/ggt243

Sato $\mathrm{H}$ (1984) Attenuation and envelope formation of three-component seismograms of small local earthquakes in randomly inhomogeneous lithosphere. J Geophys Res 89:1221-1241

Tsukada S, Odaka S, Ashiya K, Ohtake K, Nozaka D (2004) Analysis of the envelope waveform of the initial part of P-waves and its application to quickly estimating the epicentral and magnitude. Jishin 56:351-361, in Japanese with English abstract

Yoshimoto K, Sato H, Ohtake M (1997a) Three-component seismogram envelope synthesis randomly inhomogeneous semi-infinite media based on the single scattering approximation. Phys Earth Planet Inter 104:37-61

Yoshimoto K, Sato H, Ohtake M (1997b) Short-wavelength crustal heterogeneities in the Nikko area, Central Japan, revealed from the three-component seismogram envelope analysis. Phys Earth Planet Inter 104:63-73

\section{Submit your manuscript to a SpringerOpen ${ }^{\circ}$ journal and benefit from:}

- Convenient online submission

- Rigorous peer review

- Immediate publication on acceptance

- Open access: articles freely available online

- High visibility within the field

- Retaining the copyright to your article

Submit your next manuscript at $\boldsymbol{~ s p r i n g e r o p e n . c o m ~}$ 